\title{
Squeezing Alcohols into Sonoluminescing Bubbles: The Universal Role of Surfactants
}

\author{
Rüdiger Tögel, ${ }^{1}$ Sascha Hilgenfeldt, ${ }^{2}$ and Detlef Lohse ${ }^{1}$ \\ ${ }^{1}$ Department of Applied Physics and J. M. Burgers Centre for Fluid Dynamics, University of Twente, \\ 7500 AE Enschede, Netherlands \\ ${ }^{2}$ Division of Engineering and Applied Sciences, Harvard University, \\ 29 Oxford Street, Cambridge, Massachusetts 02138
}

(Received 4 August 1999)

\begin{abstract}
We conduct an experimental study of the dependence of single bubble sonoluminescence intensity on the concentration of various alcohols. The light intensity is reduced by one-half at a molar fraction of ethanol of $\approx 2.5 \times 10^{-5}$; butanol achieves the same reduction at a concentration 10 times smaller. We account for the results by a theoretical model in which the alcohols are assumed to be mechanically forced into the bubble at collapse, modifying the adiabatic exponent of the gas. The increasing hydrophobicities of the alcohols lead to decreasing effective adiabatic exponents, and thus to less heating and therefore less light. Support for this model is obtained by replotting the experimental light intensity values vs the calculated exponents, yielding a collapse of all data onto a universal curve.
\end{abstract}

PACS numbers: $78.60 . \mathrm{Mq}$

In 1990 Gaitan [1] discovered that a single air bubble trapped in an acoustical field can emit bursts of light so strong as to be visible to the naked eye. During recent years extensive theoretical and experimental research has been done in this field, and now the basic mechanisms of this so-called single bubble sonoluminescence (SBSL) seem to be resolved [2-17]. The parameter regime of SBSL is set by the shape stability of the bubble [3-5], its diffusive stability $[3,18]$, and its chemical stability $[8,12,13]$. The light seems to originate from thermal bremsstrahlung $[10,11,16,17]$, induced by (nearly) adiabatic heating of the bubble in the final stages of the radial collapses induced by the acoustic driving.

Inspired by the experimental finding that one drop of alcohol can extinguish SBSL $[19,20]$ and by the study on the effect of alcohols in multibubble sonoluminescence (MBSL) by Grieser's group [21,22], we experimentally study the influence of various alcohols on SBSL in the present paper.

A SBSL bubble was created in a spherical flask containing purified water, driven by two piezotransducers at a frequency of $35 \mathrm{kHz}$. Degassing of the dissolved air was achieved through boiling. The oxygen concentration was directly monitored through oxyometry from which the argon concentration that is the relevant one in SBSL [8] can easily be found. The light from the bubble was focused onto a high-sensitivity photodiode. The resulting photocurrent signal was amplified, digitized, and read out by a computer.

Small amounts of different alcohols (ethanol $\mathrm{C}_{2} \mathrm{H}_{5} \mathrm{OH}$, 2-propanol $\mathrm{C}_{3} \mathrm{H}_{7} \mathrm{OH}$, and 1-butanol $\mathrm{C}_{4} \mathrm{H}_{9} \mathrm{OH}$ ) were added. Subsequent stirring was necessary because of the long diffusive time scale of alcohol in water $\left(\tau_{\mathrm{diff}}=R_{\mathrm{flask}}^{2} / D \approx\right.$ 29 days with the flask radius $R_{\text {flask }}=5 \mathrm{~cm}$ and the typical diffusion constant $D \approx 10^{-5} \mathrm{~cm}^{2} / \mathrm{s}$ of the abovementioned alcohols in water). The driving frequency must be close to a resonance frequency of the flask in order to obtain high driving pressures and therefore SBSL. Adding a small liquid volume detunes the resonator, so that small adjustments of the driving frequency $(\leqslant 10 \mathrm{~Hz})$ were necessary in order to keep the driving pressure constant (at the peak of the resonance). Possible effects of this adjustment of resonance frequency were checked by adding an equal amount of water instead of alcohol. No intensity changes were observed in this case.

The light intensity as a function of the bulk molar fraction of alcohol $n_{b}$ is seen in Fig. 1. Tiny amounts of alcohol strongly decrease the intensity in all cases. The effect is more pronounced the longer the carbon chain of the alcohol: for butanol a 10 times smaller concentration is sufficient to halve the light intensity; see Table I.

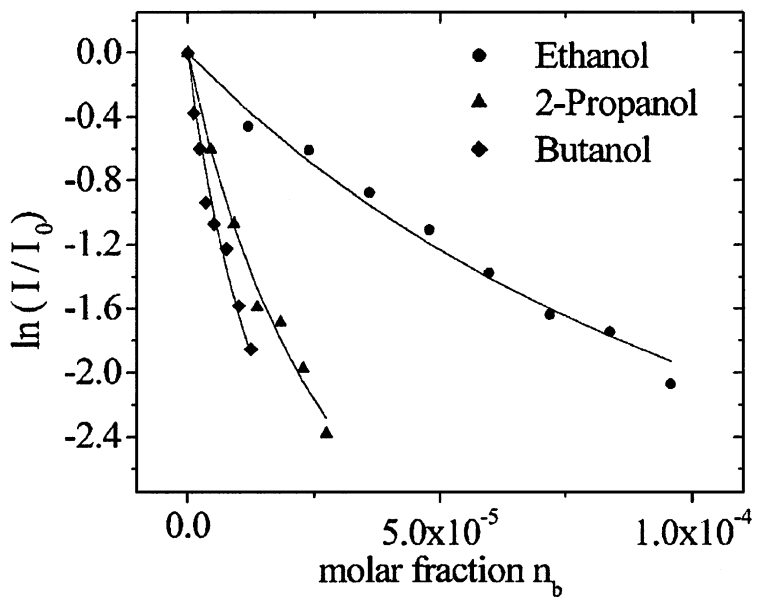

FIG. 1. Logarithmic plot of the normalized SBSL light intensities vs bulk alcohol concentration $n_{b}$. The symbols refer to measurements with different alcohol species. The solid lines show fits according to Eq. (5). Note that the dependence $I\left(n_{b}\right)$ is in general not exponential, but approaches exponential decay for very small $n_{b}$. 
TABLE I. Characteristic parameters of the intensity data. $n_{b}[I(0) / 2]$ is the measured bulk alcohol concentration at which the SL intensity is halved, $f_{\text {alc }}$ the number of degrees of freedom. $A_{\text {alc }}$ results from the fit Eq. (5) to the data and $A_{\text {alc,th }}$ from theory. $r_{\text {dif }}$ is obtained from Eq. (9). The change in surface tension with $n_{b}$ and the vapor pressure of the pure substance (last two columns) are material parameters taken from Refs. [28,29] (for a temperature of $25^{\circ} \mathrm{C}$ ).

\begin{tabular}{lccrrrrr}
\hline \hline Alcohol & $n_{b}[I(0) / 2]$ & $f_{\mathrm{alc}}$ & $A_{\mathrm{alc}}$ & $A_{\mathrm{alc}, \mathrm{th}}$ & $r_{\mathrm{dif}}$ & $\frac{\partial \sigma}{\partial n_{b}} / g s^{-2}$ & $p_{v} / k P a$ \\
\hline Ethanol & $2.6 \times 10^{-5}$ & 48 & 475 & 564 & 14.9 & -1213 & 7.87 \\
Propanol & $5.3 \times 10^{-6}$ & 66 & 1568 & 1681 & 4.2 & -3614 & 2.76 \\
Butanol & $2.7 \times 10^{-6}$ & 84 & 1920 & 4190 & 1.3 & -9007 & 0.86 \\
\hline \hline
\end{tabular}

Qualitatively, Ashokkumar et al. [21] observe the same trend in MBSL, but at considerably larger alcohol concentrations, with half-intensity concentrations of $n_{b} \approx 2 \times$ $10^{-3}$ (ethanol) and $n_{b} \approx 1 \times 10^{-4}$ (butanol). They postulate [21] that the hydrophobic alcohol molecules accumulate at the bubble surface, are then forced inside during bubble collapse, and there quench the light emission. The surface accumulation will be stronger for longer carbon chains. Our work picks up these ideas for the SBSL case with some modifications, and proceeds to develop a quantitative model. The calculation of the expected light intensity within our theory can be subdivided into four steps.

(i) Molar fraction $n_{g}\left(n_{b}\right)$ in the gas bubble. - The alcohol bulk concentration $n_{b}$ determines the molar alcohol fraction $n_{g}$ inside the bubble. This dependence is, in general, nontrivial, with hydrophobicity, vapor pressure, surface tension, and even the bubble dynamics itself factoring in [23]. For low concentrations, however, we can assume a linear dependence,

$$
n_{g}=A_{\mathrm{alc}} n_{b},
$$

with an alcohol dependent fit parameter $A_{\text {alc }}$. Later we will theoretically calculate $A_{\mathrm{alc}}$, finding good agreement with the fit results.

(ii) Adiabatic exponent $\gamma\left(n_{g}\right)$ of the alcohol-argon mixture. - After the alcohol has been squeezed into the bubble, it basically contains a mixture of argon, which accumulates in sonoluminescing air bubbles [8], and the vapor of the employed alcohol. The effective adiabatic exponent of the mixture can be derived from Dalton's law,

$$
\gamma\left(n_{g}\right)=\frac{n_{g}\left(f_{\mathrm{alc}}+2\right)+\left(1-n_{g}\right)\left(f_{\mathrm{Ar}}+2\right)}{n_{g} f_{\mathrm{alc}}+\left(1-n_{g}\right) f_{\mathrm{Ar}}} .
$$

Here, $f_{\mathrm{Ar}}=3$ and $f_{\mathrm{alc}}$ indicate the number of degrees of freedom of argon and alcohol, respectively. As temperatures inside a collapsing SBSL bubble very likely correspond to energies of 1-2 eV [16], while typical energies for vibrational quanta are $\leq 0.1 \mathrm{eV}$ (those of rotational quanta are even 2 orders of magnitude smaller), we can safely assume that all degrees of freedom are available. Thus, $f_{\mathrm{alc}}=6+2(3 N-6)$, where $N$ is the number of atoms in the molecule. Note that $\gamma$ in (2) will only slightly change in the early stages of heating of the bubble, when the alcohol molecules begin to dissociate, as $\gamma$ depends only on the product $n_{g}\left(f_{\mathrm{alc}}-3\right)$, and $f_{\mathrm{alc}} \gg 1$ : For example, dissociation in two products of about equal size roughly halves the number of degress of freedom of each but doubles $n_{g}$.

(iii) Maximum temperature $T_{\max }(\gamma)$ in the bubble.SBSL light emission has been explained satisfactorily as thermal bremsstrahlung of the (nearly) adiabatically heated gas inside the bubble $[10,11,16,17]$. Calculations coupling bubble dynamics with heat exchange have shown [24] that the gas inside the bubble maintains the ambient temperature most of the time. Only at collapse it is heated adiabatically for a brief time. To keep the model simple, we will replace the actual change in thermal coupling by an abrupt crossover from isothermal to adiabatic behavior at a certain bubble radius $R_{\text {ad }}$. From $R_{\text {ad }}$, the bubble is assumed to adiabatically collapse down to $R_{\min }$, which is very close to the van der Waals hard core radius $h$. From the van der Waals equation of state, we therefore have

$$
T_{\max }\left(n_{g}\right) \propto\left(\frac{R_{\mathrm{ad}}^{3}-h^{3}}{R_{\min }^{3}-h^{3}}\right)^{\gamma\left(n_{g}\right)-1} .
$$

As a priori we do not know the length ratio $B_{\text {alc }} \equiv$ $\left[\left(R_{\mathrm{ad}}^{3}-h^{3}\right) /\left(R_{\min }^{3}-h^{3}\right)\right]^{1 / 3}$, it is the second fit parameter of the model.

(iv) Light intensity $I\left(T_{\max }\right)$. - References $[10,11,16,17]$ show that the light intensity $I$ (as a function of the temperature) is essentially obtained as the product of two factors: (a) blackbody radiation according to the actual temperature of the bubble $\left(\propto T^{4}\right)$, and (b) the finite opacity of the bubble (dominated by a factor $\propto \exp \left(-E_{\text {ion }} / 2 k_{B} T\right), E_{\text {ion }}$ being the ionization energy of argon and $k_{B}$ the Boltzmann constant). The relative intensity reduction due to the addition of alcohol is thus approximately

$$
\begin{aligned}
\frac{I\left(n_{g}\right)}{I(0)}= & {\left[\frac{T_{\max }\left(n_{g}\right)}{T_{\max }(0)}\right]^{4} } \\
& \times \exp \left[-\frac{E_{\text {ion }}}{2 k_{B}}\left(\frac{1}{T_{\max }\left(n_{g}\right)}-\frac{1}{T_{\max }(0)}\right)\right] .
\end{aligned}
$$

We now plug the four elements of the model together and obtain a well-defined function of light intensity vs $n_{b}$,

$$
\frac{I\left(n_{b}\right)}{I(0)}=F\left(n_{b}, f_{\mathrm{alc}}, A_{\mathrm{alc}}, B_{\mathrm{alc}}\right),
$$

with two fit parameters $A_{\mathrm{alc}}$ and $B_{\mathrm{alc}}$ to which we have attributed physical meaning above. The nonlinear fit (5) indeed describes our data sets very well; see Fig. 1. Furthermore, we find that the parameter $B_{\mathrm{alc}}$ is virtually the 
same for all alcohols (it varies by about $\pm 10 \%$ around $B_{\text {alc }}=1.3$ ). It can therefore be fixed independently of the alcohol species without affecting the quality of the fit. The physical reason for this is that the radius $R_{\text {ad }}$ is determined by the external parameters like argon concentration (via $R_{0}$ ), driving pressure, etc., on which the addition of small amounts of alcohol has very little influence. Here we fixed it at $R_{\text {ad }}=1.25$. Best fits of the parameter $A_{\text {alc }}$ for $T_{\max }\left(n_{b}=0\right)=15000 \mathrm{~K}$ [16] are listed in Table I [25].

The small value of $B$ shows that adiabaticity is achieved close to $R_{\min }$, in agreement with Rayleigh-Plesset simulations [16].

The most striking result of the fit is the strong increase of $A_{\text {alc }}$ with increasing carbon chain length. This should be expected as the longer alcohols are more hydrophobic and accumulate more strongly at the gas-water interface. For the same concentration inside the bubble, and consequently the same amount of light reduction, very different alcohol bulk concentrations are needed, as observed. We also find that the required alcohol concentration $n_{g}$ inside the gas bubble for halving the intensity is always very similar, $n_{g} \approx 0.01-0.02$, regardless of the type of alcohol. This resembles the result in [21] for MBSL, where the light intensity is roughly independent of the kind of alcohol added if plotted against the alcohol surface concentration at the gas-water interface.

According to the theory presented above, the resulting light intensities are a function of both $n_{g}$ and $f_{\mathrm{alc}}$, so that one would not expect completely universal concentrations $n_{g}$. We observe, however, that after fixing $B$, the relative intensity is a unique function of the adiabatic exponent $\gamma$ only. We can thus test the theory by plotting $I$ vs $\gamma$, where all the data for the different alcohols should collapse onto one universal curve. Indeed, Fig. 2 reveals that the data, when plotted in this fashion, show a degree of universality even higher than the aforementioned dependence of MBSL intensity on surface concentration of alcohol (Fig. 8 of Ref. [21]). Figure 2 is also an a posteriori confirmation of

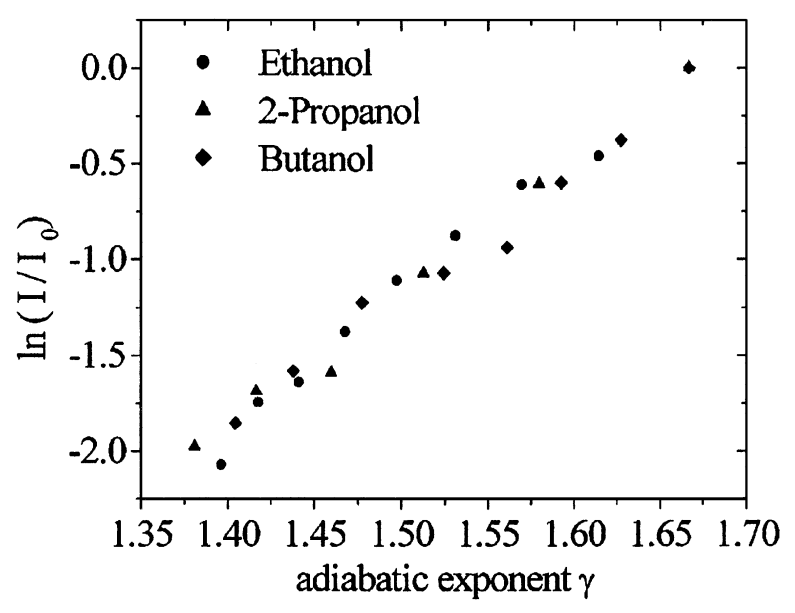

FIG. 2. Relative light intensity data as in Fig. 1, now plotted vs the effective adiabatic exponent $\gamma$ of the argon-alcohol mixture. All data collapse onto one universal curve. our assumption that the light is of essentially thermal origin and that it is the adiabatic heating of the argon-alcohol gas mixture which causes the emission.

We will now theoretically calculate the proportionality constants $A_{\text {alc }}$ in Eq. (1) and thereby get further insight into the mechanism that squeezes the alcohols into the bubble. For dilute solutions the bulk concentration $n_{b}$ of surfactant is related to its surface excess $\Gamma_{s}$ and the change in surface tension $\sigma$ via the Gibbs relation [26],

$$
\Gamma_{s}=-\frac{n_{b}}{k_{B} T}\left(\frac{\partial \sigma}{\partial n_{b}}\right)_{T} .
$$

The maximum achievable (critical) surface excess is given by close-packing of the surfactant molecules on the surface, each of which occupies an area of about $0.25 \mathrm{~nm}^{2}$ [27], thus defining $\Gamma_{s, \text { crit }}=\left(0.25 \mathrm{~nm}^{2}\right)^{-1}$. We will show below that the results of the theory do not depend on the exact area per molecule. $\Gamma_{s, \text { crit }}$ implies a critical number of alcohol molecules sitting on the interface at bubble collapse, $N_{\text {crit }}\left(R_{\text {min }}\right)=\Gamma_{s, \text { crit }} 4 \pi R_{\min }^{2}$. This number must be compared with the number of alcohol molecules which are "loaded" onto the surface at the bubble radius maximum, $N\left(R_{\max }\right)=\Gamma_{s} 4 \pi R_{\max }^{2}$. We now assume that the alcohol molecules will not desorb or diffuse away in the short time interval of bubble collapse, but that the total excess of alcohol at bubble minimum, $\Delta N=N\left(R_{\max }\right)-N_{\text {crit }}\left(R_{\min }\right)$, will enter the bubble, resulting in the alcohol concentration

$$
n_{g}=\Delta N /\left(N_{\mathrm{Ar}}+\Delta N\right)
$$

in the gas bubble. Eventually, this alcohol will be "burned" (just as the nitrogen or oxygen molecules sucked into the bubble [8]), and the reaction products will dissolve in the water.

For the forcing pressures $(\approx 1.3 \mathrm{~atm})$ and argon concentrations $(\approx 0.2 \%-0.4 \%$ of saturation) of our experiment, a typical value for the ambient radius is $R_{0}=5 \mu \mathrm{m}$ [3,4], corresponding to $N_{\mathrm{Ar}} \approx 1.67 \times 10^{10}$. Minimum and maximum radii in this regime are $R_{\min } \approx R_{0} / 10$ and $R_{\max } \approx 10 R_{0}$, respectively [7]. With $\partial \sigma / \partial n_{b}$ from Table I we get $N_{\text {crit }}\left(R_{\text {min }}\right) \approx 1.3 \times 10^{7}$, whereas in the experimental range of values for $n_{b}$ we have $N\left(R_{\max }\right) \approx$ $1.3 \times 10^{9}$ for ethanol and $N\left(R_{\max }\right) \approx 1.2 \times 10^{9}$ for butanol. In all cases, $N_{\mathrm{Ar}} \gg N\left(R_{\max }\right) \gg N_{\text {crit }}\left(R_{\min }\right)$ so that (7) simplifies to $n_{g} \approx N\left(R_{\max }\right) / N_{\mathrm{Ar}}$. Thus, the exact value of $\Gamma_{s, \text { crit }}$ does not matter, as nearly all alcohol molecules which accumulate at the bubble surface at maximum get squeezed into the bubble at collapse.

From Eqs. (6) and (7) we obtain an a posteriori justification of the assumed linear relation between $n_{g}$ and $n_{b}$ for small $n_{b}<N_{\operatorname{Ar}}\left[\frac{4 \pi R_{\max }^{2}}{k_{B} T}\left|\left(\frac{\partial \sigma}{\partial n_{b}}\right)_{T}\right|\right]^{-1}$. The desired theoretical value of $A_{\mathrm{alc}}$ is then

$$
A_{\mathrm{alc}, \mathrm{th}}=\frac{4 \pi R_{\max }^{2}}{N_{\mathrm{Ar}} k_{B} T}\left|\left(\frac{\partial \sigma}{\partial n_{b}}\right)_{T}\right| .
$$

We find good agreement with the fitted values, see Table I. As $A_{\text {alc,th }}$ depends on $R_{\max }$, the experiment was conducted 
such that for all three alcohols a bubble with $R_{0} \approx 5 \mu \mathrm{m}$ was obtained, giving roughly the same $R_{\max }$.

We stress that, as in MBSL [22], it is not the liquid vapor pressure which determines $\gamma$ : it is highest for ethanol and lowest for butanol (Table I), which would suggest ethanol as the most efficient light quenching agent.

Two crucial assumptions were made in deriving (8), which have to be checked: (i) Equation (6) is an equilibrium formula; therefore, there has to be enough time for the bubble to accumulate $N\left(R_{\max }\right)$ alcohol molecules within, say, half a driving period. (ii) The collapse must be fast enough to ensure that the excess alcohol does not diffuse back into the liquid but jumps into the bubble.

Condition (i) implies that $N_{\text {dif }}$, the number of alcohol molecules that can attach to the surface within half a cycle, be greater than $N\left(R_{\max }\right)$. We assume a diffusion-limited adsorption process and estimate $N_{\mathrm{dif}} \approx 4 \pi R_{\mathrm{max}}^{2} l_{\mathrm{dif}} n_{b} \times$ $N_{A} \rho / M$, where $N_{A}$ is Avogadro's constant, $\rho$ the density of water, $M$ its molecular mass, and $l_{\mathrm{dif}}=(D / 2 f)^{1 / 2}$ the diffusive length scale. Thus we demand

$$
r_{\mathrm{dif}} \equiv \frac{N_{\mathrm{dif}}}{N\left(R_{\max }\right)}=\left(\frac{D}{2 f}\right)^{1 / 2} \frac{G T}{\left|\left(\partial \sigma / \partial n_{b}\right)_{T}\right|} \frac{\rho}{M}>1,
$$

where $G$ is the universal gas constant. Table I shows that (9) is well fulfilled for ethanol and propanol, and is just marginally valid for butanol. The latter may account for the relatively large deviation between $A_{\mathrm{alc}}$ and $A_{\mathrm{alc}}$,th for butanol (see Table I). As surface excesses become even larger for higher alkanols, we predict deviations from this theory in experiments with, e.g., pentanol or hexanol.

To assess condition (ii) we make a worst-case estimate, disregarding the surface affinity of the alcohols, allowing them to diffuse freely during the collapse which lasts typically $\Delta t_{\text {col }} \sim 1 \mathrm{~ns}$. A typical diffusion distance is then $\sim\left(D \Delta t_{\mathrm{col}}\right)^{1 / 2} \sim 1 \mathrm{~nm}$, which is on the order of a molecule length, and implies that the alcohol cannot escape from the bubble surface during collapse.

For MBSL, Grieser and co-workers suggested that the alcohols and their reaction products accumulate in the bubble only over many cycles [22]. Such an accumulation might also occur in SBSL [20]. However, we believe that SBSL bubbles become too hot to sustain and accumulate nonsoluble molecules such as $\mathrm{C}_{2} \mathrm{H}_{2}$. A crucial experiment, to distinguish between the picture of Refs. [20,22] and the present one, would be to suddenly increase the forcing pressure of a non-SL argon bubble in the alcohol-water mixture as done in [13] for air and argon bubbles in pure water: If accumulative processes over many cycles play a role, the bubble should still glow brightly for some period of time, in spite of the alcohol.

It is our pleasure to acknowledge stimulating discussions with F. Grieser, S. Grossmann, T. Matula, A. Prosperetti, and K. Suslick. The work is part of the research program of FOM, which is financially supported by NWO.
[1] D.F. Gaitan, Ph.D. thesis, The University of Mississippi, 1990.

[2] L. A. Crum, Phys. Today 47, No. 9, 22 (1994).

[3] S. Hilgenfeldt, D. Lohse, and M. P. Brenner, Phys. Fluids 8, 2808 (1996).

[4] R. G. Holt and D. F. Gaitan, Phys. Rev. Lett. 77, 3791 (1996).

[5] A. Prosperetti, Q. Appl. Math. 34, 339 (1977); M. Brenner, D. Lohse, and T. Dupont, Phys. Rev. Lett. 75, 954 (1995); A. Prosperetti and Y. Hao, Philos. Trans. R. Soc. London 357, 203 (1999).

[6] V. Q. Vuong and A. J. Szeri, Phys. Fluids 8, 2354 (1996).

[7] B. P. Barber et al., Phys. Rep. 281, 65 (1997).

[8] D. Lohse et al., Phys. Rev. Lett. 78, 1359 (1997); D. Lohse and S. Hilgenfeldt, J. Chem. Phys. 107, 6986 (1997).

[9] B. Gompf et al., Phys. Rev. Lett. 79, 1405 (1997); R. Pecha, B. Gompf, G. Nick, Z. Q. Wang, and W. Eisenmenger, Phys. Rev. Lett. 81, 717 (1998).

[10] W. Moss, D. Clarke, and D. Young, Science 276, 1398 (1997); W. C. Moss et al., Phys. Rev. E 59, 2986 (1999).

[11] L. Frommhold, Phys. Rev. E 58, 1899 (1998).

[12] J. A. Ketterling and R. E. Apfel, Phys. Rev. Lett. 81, 4991 (1998).

[13] T. J. Matula and L. A. Crum, Phys. Rev. Lett. 80, 865 (1998).

[14] R. Apfel, Nature (London) 398, 378 (1999).

[15] F. Gaitan, Phys. World 12, 20 (1999).

[16] S. Hilgenfeldt, S. Grossmann, and D. Lohse, Nature (London) 398, 402 (1999); Phys. Fluids 11, 1318 (1999).

[17] K. Yasui, Phys. Rev. E 60, 1754 (1999).

[18] M. M. Fyrillas and A. J. Szeri, J. Fluid Mech. 277, 381 (1994).

[19] K. R. Weninger et al., J. Phys. Chem. 99, 14195 (1995).

[20] M. Ashokkumar et al., in Proceedings the 16th International Congress on Acoustics and 135th Meeting of the Acoustical Society of America, Seattle, WA, 1998 (The Society, Woodbury, NY, 1998), Vol. 3, p. 1543; T. Matula, in Proceedings of the International Symposium on Nonlinear Acoustics, Göttingen, 1999 (to be published).

[21] M. Ashokkumar, R. Hall, P. Mulvaney, and F. Grieser, J. Phys. Chem. 101, 10845 (1997).

[22] M. Ashokkumar, P. Mulvaney, and F. Grieser, J. Am. Chem. Soc. 121, 7355 (1999).

[23] M. M. Fyrillas and A. S. Szeri, J. Fluid Mech. 311, 361 (1996).

[24] M. Plesset and A. Prosperetti, Annu. Rev. Fluid Mech. 9, 145 (1977).

[25] In principle, $T_{\max }\left(n_{b}=0\right)$ could be considered a (third) fit parameter. It is, however, known at least in principle from the theory without alcoholic surfactants [16]. Also, both $A_{\text {alc }}$ and $B_{\text {alc }}$ show only a weak dependence on changes in $T_{\max }(0)$ : varying $T_{\max }(0)$ between 15000 and $30000 \mathrm{~K}$, $B_{\text {alc }}$ changes by $14 \%$ and $A_{\text {alc }}$ by only $1 \%$.

[26] P. W. Atkins, Physical Chemistry (Oxford University Press, Oxford, 1995).

[27] G. L. Gaines, Insoluble Monolayers at Liquid/Gas Interfaces (Interscience: John Wiley, New York, 1966).

[28] Handbook of Chemistry, edited by N. A. Lange (McGrawHill, New York, 1961).

[29] D. R. Lide, Handbook of Chemistry and Physics (CRC Press, Boca Raton, 1991). 